\title{
Erratum to: The linkup of mono-elemental solutions to the SI using INAA: a measurement procedure and the achievable uncertainty
}

\author{
Giancarlo D'Agostino' $^{1}$ - Luigi Bergamaschi ${ }^{1}$ - Marco Di Luzio ${ }^{1,2}$. \\ Janine Noordmann ${ }^{3} \cdot$ Massimo Oddone $^{2}$ - Olaf Rienitz ${ }^{3}$
}

Published online: 19 June 2017

(C) Akadémiai Kiadó, Budapest, Hungary 2017

Erratum to: J Radioanal Nucl Chem (2016)

$$
\begin{aligned}
& \text { 309:777-786 } \\
& \text { DOI 10.1007/s10967-015-4676-2 }
\end{aligned}
$$

The formula S1 included in the Electronic Supplementary Material was published incorrectly. The correct formula is provided below.

$\sqrt{\frac{C_{\mathrm{rsu}}\left(t_{\mathrm{d}}\right)}{C_{\mathrm{usd}}\left(t_{\mathrm{d}}\right)}}=\frac{1-\frac{\Delta d}{d}}{1+\frac{\Delta d}{d}}$,

Electronic supplementary material The online version of this article (doi:10.1007/s10967-017-5307-x) contains supplementary material, which is available to authorized users.

The online version of the original article can be found under doi:10.1007/s10967-015-4676-2.

Giancarlo D'Agostino

g.dagostino@inrim.it

1 Istituto Nazionale di Ricerca Metrologica (INRIM) - Unit of

Radiochemistry and Spectroscopy, c/o Department of

Chemistry, University of Pavia, Via Taramelli 12,

27100 Pavia, Italy

2 Department of Chemistry - Radiochemistry Area, University of Pavia, Via Taramelli 12, 27100 Pavia, Italy

3 Physikalisch-Technische Bundesanstalt (PTB), Bundesallee 100, 38116 Brunswick, Germany 